ROCHA, R.C. et al. Sopro intermitente em cão: relato de caso. PUBVET, Londrina, V. 8, N. 17, Ed. 266, Art. 1771, Setembro, 2014.

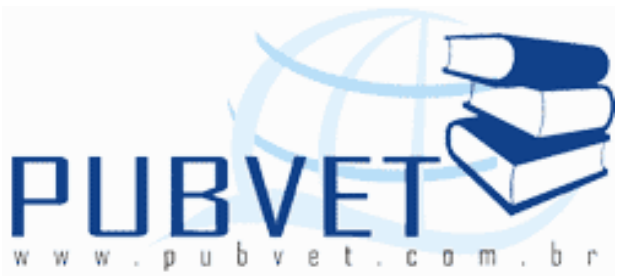

PUBVET, Publicações em Medicina Veterinária e Zootecnia.

\title{
Sopro intermitente em cão: relato de caso
}

Rogério Carletti Rocha ${ }^{1}$, Cesar Martins de Souza ${ }^{2}$, Rafael Teixeira Rolan ${ }^{3}$, Gabriela Santana Zebiani ${ }^{4}$, Carlos Alberto Sanchez ${ }^{4}$, Robson Oliveira Santos ${ }^{4}$

${ }^{1}$ Graduando Medicina Veterinária pela Universidade Guarulhos / SP;

2 Pós-graduando pelo programa de Residência em Clínica Médica de Pequenos Animais pelo Hospital Veterinário Hovet - Universidade Guarulhos; Pósgraduando em Cardiologia Veterinária pela Anclivepa - SP

3 Docente, Unidade Acadêmica de Medicina Veterinária, Universidade Guarulhos;

${ }^{4}$ Graduandos Medicina Veterinária pela Universidade Guarulhos / SP.

\section{Resumo}

O prolapso valvar é uma condição decorrente da degeneração das cordas tendíneas e folhetos valvares, que impedem a valva acometida de realizar sua função de fechamento adequadamente, causando um refluxo sanguíneo durante a sístole ventricular. Este refluxo levará à alterações sistêmicas secundárias, como hipertensão pulmonar, edema pulmonar e insuficiência cardíaca congestiva, se não tratada adequadamente. À ascultação, o som emitido pelo coração, quando há presença de prolapso valvar é denominado "sopro", o qual pode ser classificados em graus de um a seis. O presente trabalho objetivou relatar o caso do animal Mike, canino, Shih Tzu, macho, três anos de idade, atendido na Clínica Escola Veterinária da Universidade 
ROCHA, R.C. et al. Sopro intermitente em cão: relato de caso. PUBVET, Londrina, V. 8, N. 17, Ed. 266, Art. 1771, Setembro, 2014.

Guarulhos em 2012. Como histórico, as principais queixas eram de prostração e um episódio recente de síncope, e ruídos respiratórios quando o animal se agitava. Ao exame físico o animal apresentou um sopro valvar, com foco em valva mitral, de grau IV - VI, porém de caráter intermitente. Esta condição incomum é denominada como "Sopro Intermitente".

Palavras-chave: Prolapso valvar, Prolapso de Mitral, Sopro Intermitente

\title{
Flashing dog murmur: a case report
}

\begin{abstract}
The valve prolapse is a condition resulting from degeneration of tendinae chords and valve leaflets, which prevent the affected valve to perform its function closing properly, causing blood reflux during ventricular sistol. This reflux will lead to secondary systemic changes, such as pulmonary hypertension, pulmonary edema and congestive heart failure, if not treated properly. In auscultation, a cardiac sound is emmited when there is valve prolapse it called "murmur", which can be classified into grades from one to six. This study aims to report the case of Mike, dog, Shih Tzu, male, 3 years old, antended at Hovet Ung in 2012. As history, the main complaints were of prostration and an recent episode of syncope, and respiratory noises when excited. On physical examination the animal had a murmur, mitral valve focused, grade IV - VI, however, it was not constant. This uncommom condition is known as "Intermitent Murmur".
\end{abstract}

Keywords: Valvar Prolapse, Mitral Prolapse, Intermittent murmur.

\section{Introdução:}

O coração é o órgão responsável pelo bombeamento do sangue para as artérias e veias, através de contrações ritmadas, para todas as estruturas do corpo com o objetivo de levar o sangue com todos os nutrientes e oxigênio necessários, além do transporte de células do sistema imunológico. 
ROCHA, R.C. et al. Sopro intermitente em cão: relato de caso. PUBVET, Londrina, V. 8, N. 17, Ed. 266, Art. 1771, Setembro, 2014.

Anatomicamente, o coração é dividido em quatro câmaras: dois átrios e 2 ventrículos (Esquerdo e direito). Para que o fluxo sanguíneo ocorra unidirecionalmente durante os batimentos cardíacos, existem estruturas, com origem endotelial denominadas valvas cardíacas. São elas: Valva aórtica, valva pulmonar, valva mitral e valva tricúspide.

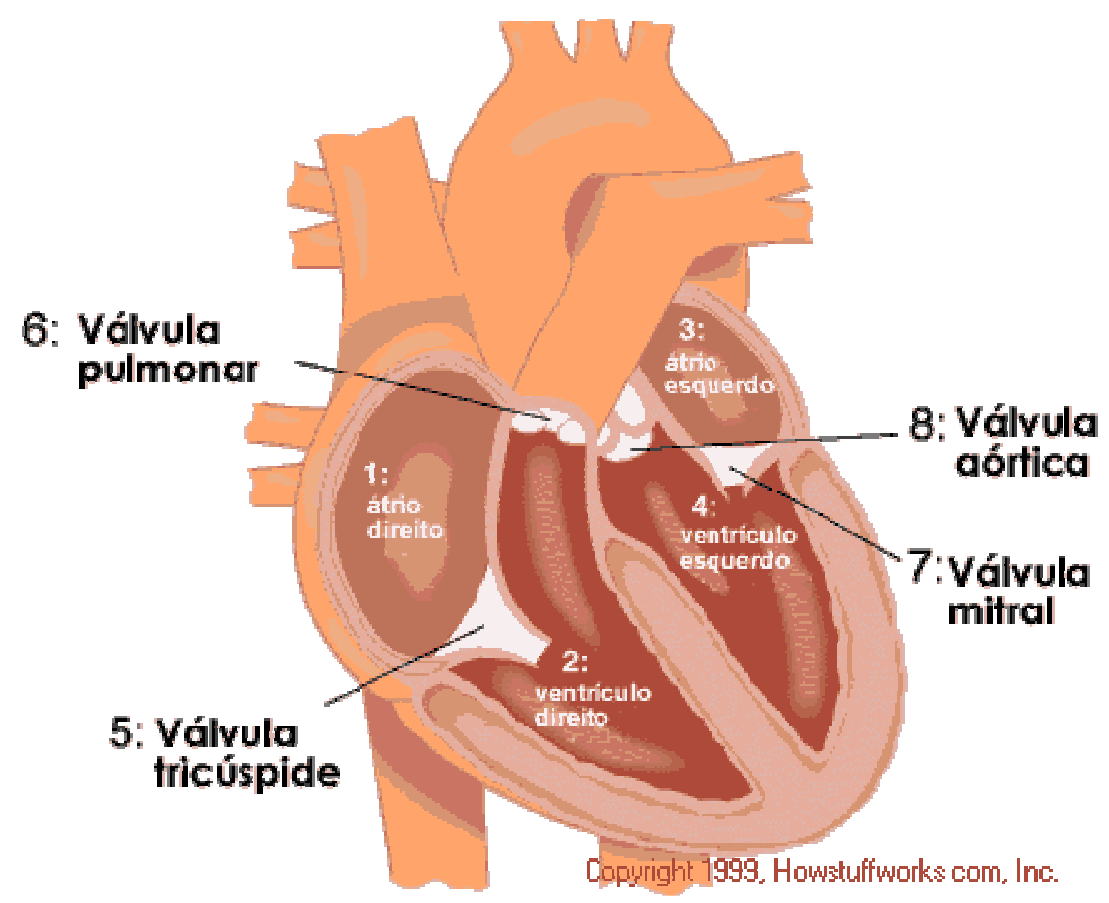

Figura 1: Camaras e Valvas Cardíacas.

A doença valvar crônica degenerativa átrio ventricular (DVCD), ou endocardiose, é apontada como causa mais comum de insuficiência cardíaca em cães. Na maioria dos casos, a valva afetada é a valva mitral, porém, alguns cães apresentam ambas as valvas atrioventriculares afetadas [1]. Apenas em $30 \%$ dos casos de doença valvar crônica degenerativa, a valva tricúspide é a afetada. Esta doença é aproximadamente 1.5 vezes mais comum em cães, do que em cadelas, e sua prevalência é maior em cães de menor porte $(<20 \mathrm{Kg}$ ), do que em cães de grande porte [14]. Geralmente os animais adultos e idosos, 
ROCHA, R.C. et al. Sopro intermitente em cão: relato de caso. PUBVET, Londrina, V. 8, N. 17, Ed. 266, Art. 1771, Setembro, 2014.

entre 8 a 11 anos. A doença é idêntica tanto em machos quanto em fêmeas, porém, em machos observa-se uma maior frequência, maior gravidade além de progressividade mais rápida. As raças que mais apresentam a DVCD são: Poodle toy, Schnauzer miniatura, Chihuahua, Pincher, Fox terrier, Boston terrier, Cocker Spaniel Inglês e Americano, Whippet e Cavalier King Charles Spaniel. $[1,11,12,13]$

Estudos patológicos comprovaram que a maioria dos cães desenvolvem a DVCD com o envelhecimento[2]. Uma das principais causas da DVCD é a degeneração mixomatosa progressiva da valva mitral, ou das duas valvas atrioventriculares em conjunto[8,9], atingindo os folhetos valvares e as cordas tendíneas [2], impedindo a coaptação completa dos folhetos, e causando consequentemente uma regurgitação valvular [10]. O Prolapso da valva mitral (MVP) é uma patologia valvar multifatorial, que pode ser causada por anormalidades no tecido valvar, incongruências geométricas entre o ventrículo e a valva, ou falhas nos tecidos de conexão valvar. O espessamento valvar, também conhecido como degeneração mixomatosa, é a causa mais comum, e é considerada a condição predisponente mais importante nos casos clínicos de MVP [3].

O Prolapso da válvula mitral (MVP) é uma protrusão anormal dos folhetos da válvula mitral, durante a sístole, em direção ao átrio esquerdo. Comumente, esta condição desenvolve uma insuficiência mitral grave[4, 5, 14, 17, 19], como demonstrado na imagem abaixo. 
ROCHA, R.C. et al. Sopro intermitente em cão: relato de caso. PUBVET, Londrina, V. 8, N. 17, Ed. 266, Art. 1771, Setembro, 2014.

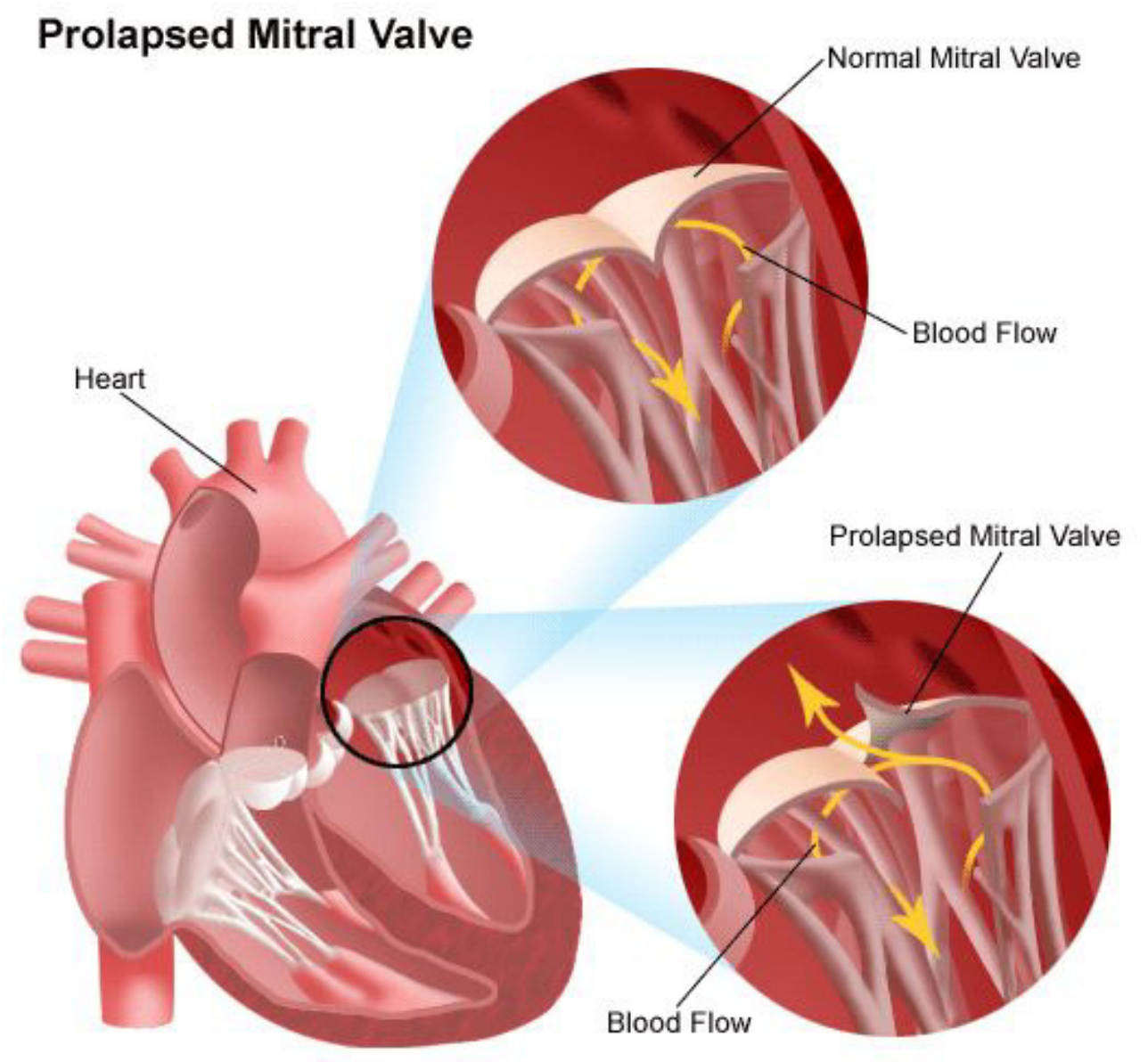

Imagem 2: Valva Mitral Prolapsada.

As lesões na valva se iniciam com pequenos nódulos em suas margens livres, que se tornam placas maiores e coalescentes que distorcem a estrutura valvar. Simultaneamente, as cordas tendíneas também são comprometidas, tornandose espessadas e fracas. Os folhetos acometidos tornam-se espessados, de forma que o tecido excedente forma uma saliência que durante a sístole se prolapsa em direção ao átrio. A partir deste prolapso, a valva, gradualmente, começa a regurgitar sangue, e conforme as lesões progridem, de forma crônica, a insuficiência valvar torna-se clinicamente evidente. Em casos avançados, alguns folhetos podem se apresentar evidentemente deformados, espessados e possivelmente retraídos [1]. 
ROCHA, R.C. et al. Sopro intermitente em cão: relato de caso. PUBVET, Londrina, V. 8, N. 17, Ed. 266, Art. 1771, Setembro, 2014.

Para realizar sua função regular, a valva mitral, necessita primariamente que sua morfologia esteja íntegra. Em segundo lugar, deve haver uma interação coordenada dos folhetos valvares, cordoalhas tendíneas, músculos papilares, e da parede do ventrículo esquerdo, para que não ocorra o prolapso na sístole ventricular[6, 7, 8,14$]$. Havendo falhas em qualquer um destes mecanismos, pode haver refluxo sanguíneo do ventrículo para o átrio, de forma a prejudicar o fluxo unidirecional regular da circulação intra cardíaca.

Mecanismos compensatórios aumentam o volume sanguíneo e o tamanho do coração (Dilatação do ventrículo e o átrio, hipertrofia miocárdica excêntrica) e permitem que a maioria dos cães permaneça assintomática por um período prolongado. Os mecanismos neuro hormonais (Sistema Nervoso Autônomo Simpático e Sistema Renina Angiotensina Aldosterona, além da liberação do Hormônio Anti Diurético (ADH)) atuam no remodelamento cardíaco [14, 17]. De início, a ativação desses sistemas auxilia a circulação, quando houver hipotensão e diminuição do volume de sangue ejetado, devido à regurgitação, aumentando a volemia através da retenção de sódio e água, devido à Aldosterona, e através da vasoconstrição acionada pela Angiotensina, favorecendo dessa forma a perfusão tecidual $[15,16]$. O Sistema Nervoso Autônomo Simpático proporciona alterações como aumento da frequência cardíaca e maior contratilidade, visando assim normalizar a volemia e débito cardíaco, diminuídos devido à regurgitação sanguínea causada pelo prolapso valvar.

Porém, a ativação destes mecanismos de forma crônica acelera o processo de decréscimo da função cardíaca, além de proporcionar alterações sistêmicas consideráveis. A retenção de volume excessiva causada pela ação da aldosterona e do $\mathrm{ADH}$, consequentemente acumulando sódio, causam edema pulmonar e em membros, ascite, congestão e efusões $[15,16]$, a vasoconstrição crônica causada pela Angiotensina aumenta a pós - carga do coração, reduz o débito cardíaco além de proporcionar um maior refluxo valvar. Alguns sinais observados são cansaço, intolerância ao exercício, distrição 
ROCHA, R.C. et al. Sopro intermitente em cão: relato de caso. PUBVET, Londrina, V. 8, N. 17, Ed. 266, Art. 1771, Setembro, 2014.

respiratória e taquicardia. À auscultação pode-se evidenciar ritmo de galope, arritmias e sopros [16].

As alterações proporcionadas pela Ativação do Sistema Nervoso Autônomo Simpático de forma crônica afetam diretamente a função cardíaca, pois promovem o aumento da pós-carga através de maior esforço cardíaco, promovendo um maior requerimento de oxigênio por parte do miocárdio, contribuindo para a ocorrência de dano celular e fibrose miocárdica, predispondo o animal a apresentar arritmias cardíacas.

O feedback necessário para que estes mecanismos (Neurológico e Hormonal) normalizem suas ações é proporcionado pelos baroreceptores arteriais e atriais. Devido ao quadro de insuficiência cardíaca causado pelo prolapso valvar, estes receptores se apresentam com uma responsividade maior, consequentemente estimulando a ativação dos sistemas compensatórios de forma crônica, e diminuindo a resposta inibitória vagal.

Já o mecanismo compensatório hormonal (Sistema Renina Angiotensina Aldosterona), promove alterações como maior retenção de água e sais, devido à ação da Aldosterona, que proporciona a reabsorção de sódio e cloreto, além da secreção de potássio e hidrogênio para os túbulos coletores, visando aumentar o volume vascular. A aldosterona também participa dos processos de inflamação e fibrose, portanto, a exposição crônica a este hormônio contribui para o processo de remodelamento patológico e fibrose miocárdica. $\mathrm{O} A D H$ também promove retenção de líquidos, além de proporcionar vasoconstrição.

A renina é produzida nos rins, e atua facilitando a conversão do angiotensinogenio, em Angiotensina I, a qual é uma forma inativa, que sofrerá a ação da Enzima Conversora de Angiotensina (ECA), e se transformará em Angiotensina II, um potente vasoconstritor e estimulante da liberação de ADH. A angiotensina II também é produzida no coração, onde influenciará diretamente nas estruturas e no funcionamento do coração, por proporcionar maior ação dos efeitos simpáticos, promovendo também alterações no tecido cardíaco, como remodelamento, hipertrofia, inflamação e fibrose [15]. 
ROCHA, R.C. et al. Sopro intermitente em cão: relato de caso. PUBVET, Londrina, V. 8, N. 17, Ed. 266, Art. 1771, Setembro, 2014.

Pode haver um grande aumento do átrio esquerdo, antes que apareça qualquer manifestação de insuficiência cardíaca; Alguns cães nunca apresentam sinais clínicos de insuficiência cardíaca. A velocidade com que a regurgitação piora, bem como o grau de distensibilidade atrial e de contratilidade ventricular, influencia a capacidade de tolerância do animal à doença [1].

O diagnóstico da DVCD se dá primariamente através da realização de ecodopplercardiograma. A ecocardiografia é o método de exame de imagem principal, caracterizado como padrão ouro para a avaliação do fluxo cardíaco, com o objetivo de diagnosticar as doenças cardíacas [20]. A introdução recente do Ecodopplercardiograma na medicina veterinária tem auxiliado muitos veterinários a diagnosticar precocemente a DVCD, de forma não invasiva [21]. A eco cardiografia permite a avaliação da atividade cardíaca em tempo real. Pode - se definir que a eco cardiografia é um método ecográfico ou ultrassonográfico do coração, realizado através de um transdutor, que emite ondas de som de alta frequência, que atravessa o tecido cardíaco, e retorna para o transdutor, gerando uma imagem visível em um monitor [19], como demonstrado na figura 3. 
ROCHA, R.C. et al. Sopro intermitente em cão: relato de caso. PUBVET, Londrina, V. 8, N. 17, Ed. 266, Art. 1771, Setembro, 2014.

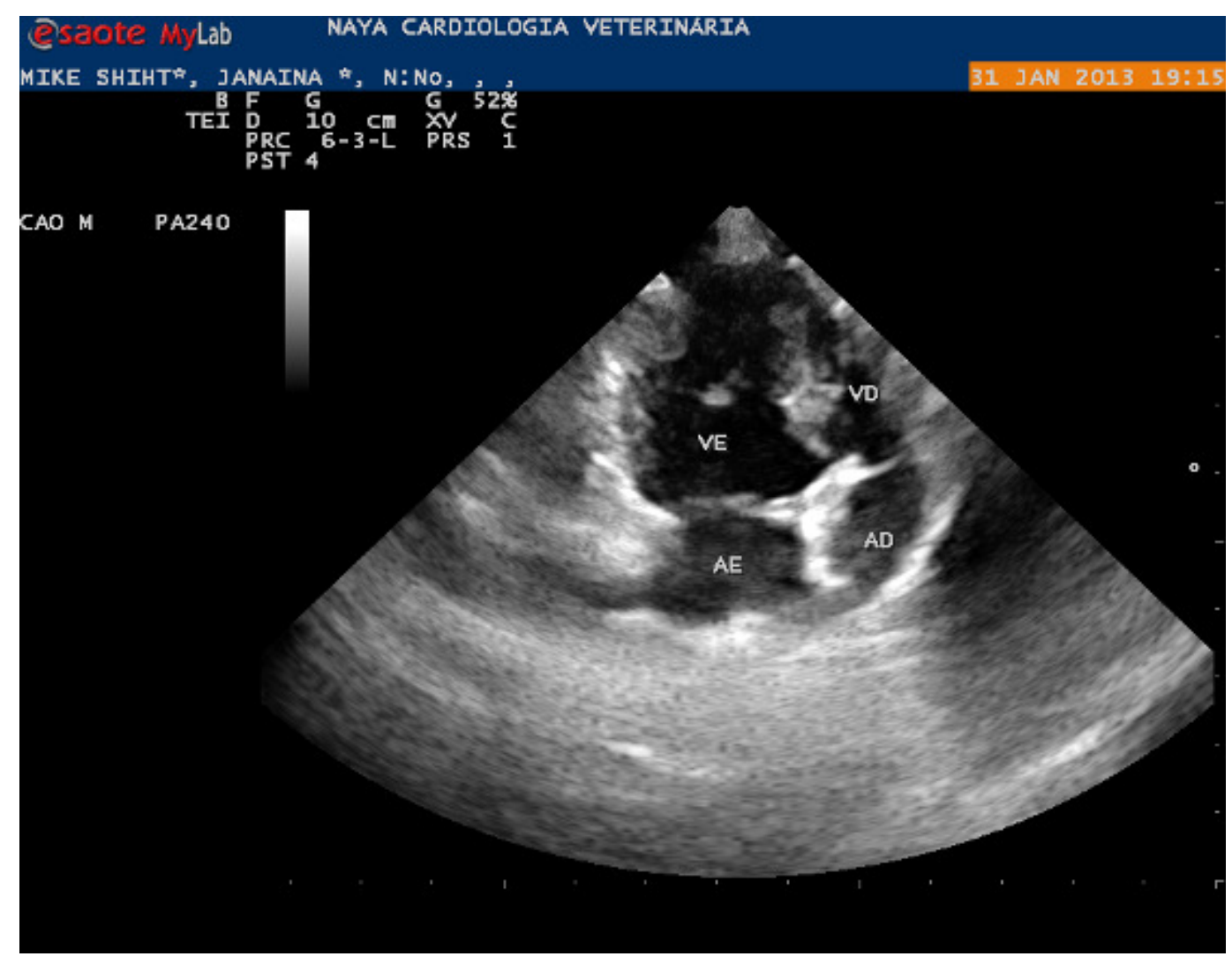

Figura 3: Camaras Cardíacas evidenciadas por Ecodopplercardiograma.

A ecocardiografia com Doppler (Ecodopplercardiograma) permite a visualização do fluxo sanguíneo, evidenciando qualquer fluxo anormal durante o ritmo cardíaco. É extremamente importante para o diagnóstico precoce do prolapso valvar, visto que o refluxo causado pelo fechamento valvar incompleto, é evidenciado durante a realização do exame, independentemente do seu grau [15]. Pode - se observar o prolapso valvar e o refluxo sanguíneo, nas figuras 4 e 5 . 
ROCHA, R.C. et al. Sopro intermitente em cão: relato de caso. PUBVET, Londrina, V. 8, N. 17, Ed. 266, Art. 1771, Setembro, 2014.

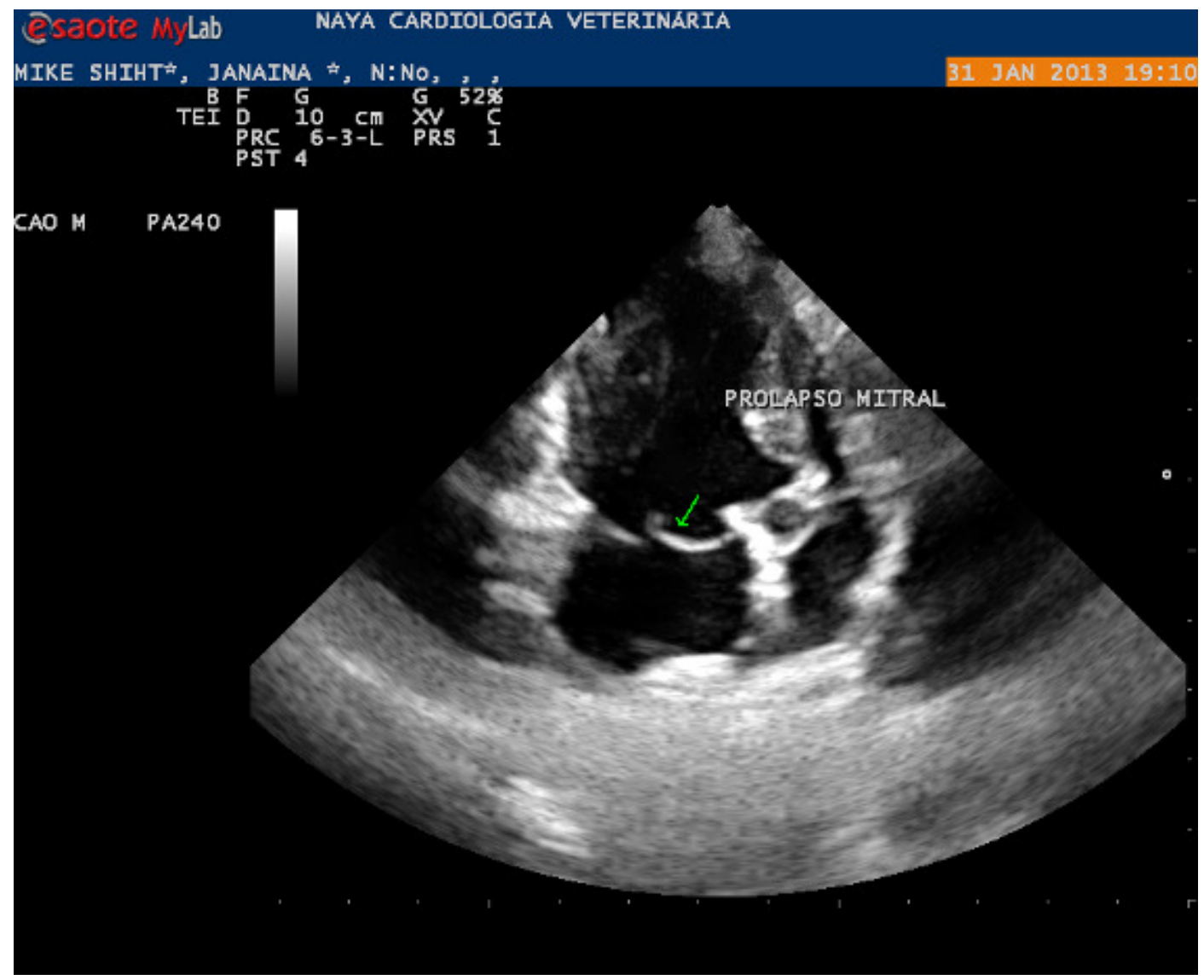

Figura 4: Prolapso Valvar evidenciado em Ecodopplercardiograma. 


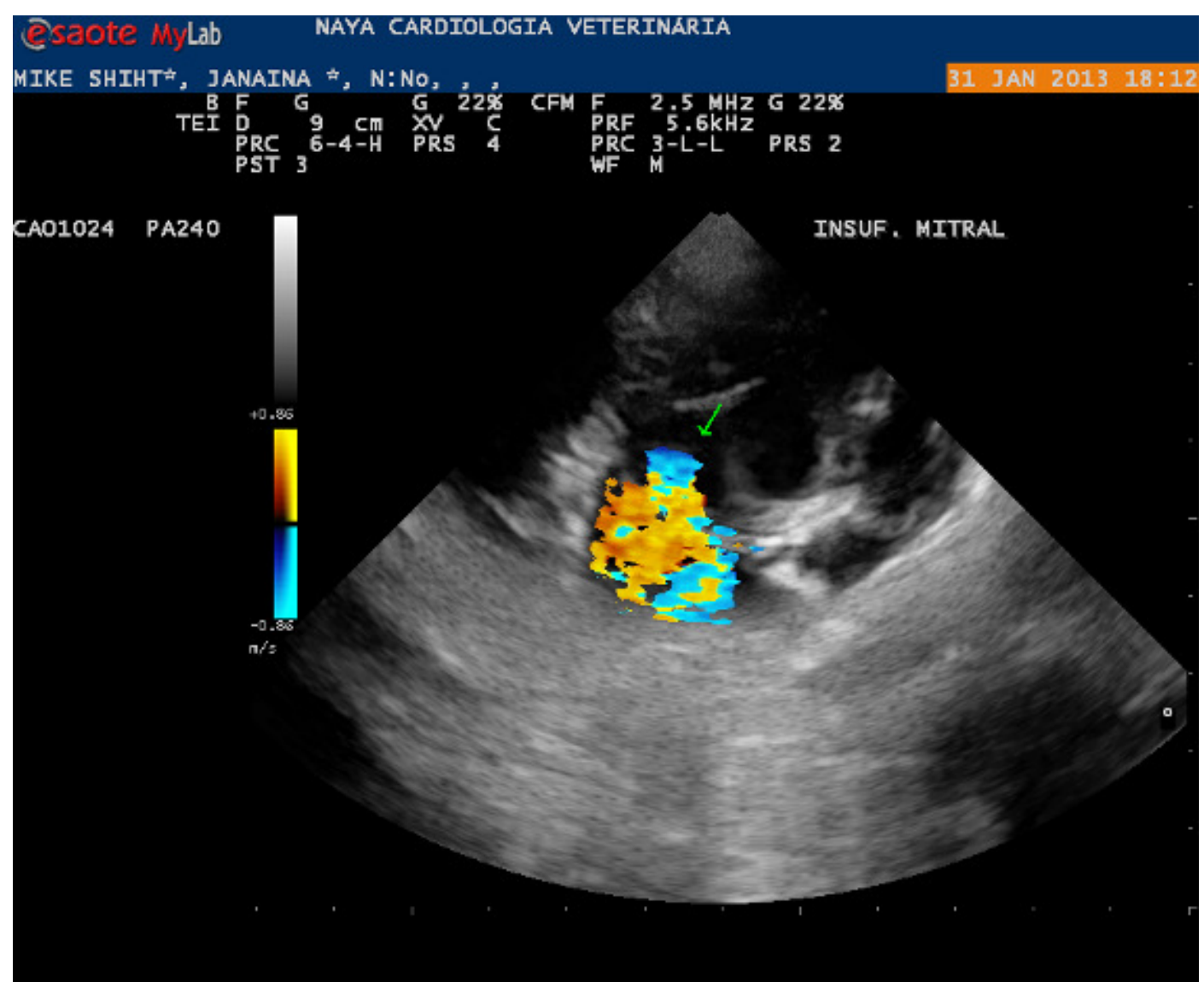

Figura 5: Mosaico em átrio esquerdo causado pelo refluxo sanguíneo proveniente do prolapso valvar.

Outro método utilizado em casos de prolapso valvar é o Raio - $X$ torácico. $O$ prolapso valvar, a longo prazo, proporciona um aumento de câmara cardíaca, devido à ação dos mecanismos compensatórios já citados. Este aumento se torna visível através do Raio - $X$ torácico, porém, este método de imagem só auxilia no diagnóstico de doença valvar quando esta já é crônica [15, 21], contudo, este método auxilia no diagnóstico de doenças pulmonares, como por exemplo quando o animal apresenta como principal sinal clínico tosse, porém esta não é de base cardíaca, e sim pulmonar. Apesar de o remodelamento de camara cardíaca, neste caso, o átrio esquerdo, ser um fator desencadeante de tosse, existem muitos outros fatores extra - cardíacos desencadeantes de tosse, como por exemplo animais com Pneumonia [24]. Além dos métodos de 
ROCHA, R.C. et al. Sopro intermitente em cão: relato de caso. PUBVET, Londrina, V. 8, N. 17, Ed. 266, Art. 1771, Setembro, 2014.

imagem, clinicamente, também é possível o diagnóstico do prolapso valvar, através da auscultação cardíaca. A auscultação Cardíaca serve para identificar sons cardíacos normais e / ou anormais, frequência e presença ou não de arritmias cardíacas [15]. Os sons mais facilmente auscultados são as bulhas (primeira e segunda), sopros de ejeção, estalidos sistólicos, abertura de estenose de valva mitral ou tricúspide, sopros diastólicos da regurgitação aórtica e pulmonar, e sopro sistólico da comunicação interventricular [18]. O som característico, causado pelo prolapso valvar, é o Sopro. Existe uma escala, designada para definir os graus do sopro, de acordo com as características do som que ele produz durante a auscultação. Esta classificação pode ser observada no quadro abaixo $[18,19]$ :

Quadro 1:

\begin{tabular}{|l|l|}
\hline Grau 1 / 6: & $\begin{array}{l}\text { Sopro de baixa intensidade, muito tênue, audível apenas } \\
\text { após alguns minutos de auscultação, em ambientes } \\
\text { silenciosos. }\end{array}$ \\
\hline Grau 2 / 6: & $\begin{array}{l}\text { Sopro ainda de baixa intensidade, porém, facilmente audível } \\
\text { com a colocação do estetoscópio sobre o ponto de foco } \\
\text { do ruído. }\end{array}$ \\
\hline Grau 3 / 6: & $\begin{array}{l}\text { Sopro de intensidade moderada, facilmente audível, com } \\
\text { propagação para outros pontos de auscultação cardíaca. }\end{array}$ \\
\hline Grau 4 / 6: & $\begin{array}{l}\text { Sopro intenso, com propagação para outras áreas de } \\
\text { Ausculta. }\end{array}$ \\
\hline Grau 5 / 6: & $\begin{array}{l}\text { Sopro bastante intenso, podendo ser auscultado com } \\
\text { facilidade, produzindo frêmito palpável. }\end{array}$ \\
\hline Grau 6 / 6: & $\begin{array}{l}\text { Sopro de maior intensidade, sendo auscultável apenas com } \\
\text { a aproximação da borda do estetoscópio ao tórax do } \\
\text { paciente, e que produz um frêmito facilmente palpável. }\end{array}$ \\
\hline
\end{tabular}

A respeito do tratamento, atualmente, há maior discussão sobre o momento em que se deve iniciar o tratamento. Em 2009 , um consenso sobre o a DVCD foi realizado, onde foram definidos estágios da doença, de acordo com a condição clínica que o animal apresenta, decorrente desta condição.

Este consenso definiu sete estágios que serão listados abaixo. 
ROCHA, R.C. et al. Sopro intermitente em cão: relato de caso. PUBVET, Londrina, V. 8, N. 17, Ed. 266, Art. 1771, Setembro, 2014.

- Classe A: Este estágio compreende os animais que possuem alto risco de desenvolver a doença, mas que ainda não possuem nenhum sinal de disfunção cardíaca ou valvar. Estes animais são aqueles que apresentam doenças como hipertensão, animais idosos, e animais predispostos a desenvolverem a doença (Exemplo: Cavalier King Charles Spaniel). Neste estágio, o consenso concluiu que não há necessidade de tratamento.

- Classe B: São os animais que possuem algum sinal ou disfunção cardíaca, mas que ainda não apresentam sintomas de Insuficiência Cardíaca Congestiva. Este estágio possui duas subdivisões, são elas:

- Classe B1: São os animais que não apresentam alterações em camaras cardíacas, causadas pela DVCD, diagnosticadas através de exames como o Ecodopplercardiograma e o Raio-X. Neste estágio ainda não há recomendação de tratamento, pois apesar da doença seguir um curso progressivo, nenhum tratamento mostrou - se eficaz na tentativa de diminuir esta progressão.

- Classe B2: São os animais que apresentam uma regurgitação valvar, sem sinais clínicos, porém, que apresentam remodelamento de camara cardíaca.

Estudos dizem que os indicadores da evolução da doença, como o tamanho das camaras cardíacas e os sinais clínicos, evoluem de uma maneira não linear, ao longo do tempo. Cães com remodelamento cardíaco causado pelo prolapso de mitral apresentam alteração hemodinâmica, em comparação com os cães que apresentam o coração com dimensões normais.

Neste estágio, o consenso indicou que tratamento com fármacos das classes IECA, Antagonistas dos Receptores Beta Adrenérgicos, Inodilatadores e bloqueadores dos receptores de mineralocorticoides podem ser benéficos.

Neste estágio, não houve consenso sobre o tratamento preventivo a base de IECA. Foram realizados dois trabalhos, VETPROOF (Veterinary Enalapril Trial to Prove Reduction in Onset of Failure), e SVEP (Scandinavian Veterinary Enalapril Prevention (Trial)). Estes trabalhos visaram avaliar a eficácia do tratamento com IECA, quando utilizado com o objetivo de prevenir o desenvolvimento de sinais clínicos da regurgitação sanguínea, causada pelo prolapso valvar, em animais assintomáticos, que apresentam a DVCD. Os estudos realizados pelo 
ROCHA, R.C. et al. Sopro intermitente em cão: relato de caso. PUBVET, Londrina, V. 8, N. 17, Ed. 266, Art. 1771, Setembro, 2014.

VETPROOF e SVPE não evidenciaram diminuição no progresso da doença, quando comparados os grupos (Grupo tratado e grupo placebo). Porém, após uma sub - análise, realizada com 96 animais, que foram analisados até sua morte, concluiu - se que a longo prazo, o tratamento preventivo com IECA apresentou resultados positivos, visto que muitos animais se apresentavam livres de Insuficiencia Cardíaca. Porém, neste consenso, não houve acordo sobre a realização de tratamento preventivo nos animais deste estágio.

- Classe C: Neste estágio encontram - se os animais que possuam sinais clínicos de falha cardíaca, como intolerância ao exercício e sincope, associados com o remodelamento, sejam eles recentes ou não. Como existem diferenças importantes em relação ao tratamento realizado em animais com insuficiência cardíaca aguda, que necessitam de suporte hospitalar, e os que podem ser tratados em casa, este estágio foi subdividido em Estágio C1 e Estágio C2.

- Classe C1: Neste estagio estão os animais que apresentam sinais de insuficiência cardíaca aguda, secundárias ao prolapso valvar ou DVCD, e necessitam de tratamento hospitalar intensivo. A crise pode ocorrer devido algum fator desencadeante, como por exemplo ruptura de cordoalha tendínea maior, excesso de fluidoterapia ou exercício exagerado. 0 tratamento nesta fase baseia - se em estabilizar a condição hemodinamica do animal, regulando a pré carga, pós carga, débito cardíaco e contratilidade.

O tratamento eleito para este estágio, conceituado pelo consenso, se dá através do uso de Furosemida injetável, para melhorar a condição respiratória do paciente. O uso de fármacos IECA, pode ser benéfico quando usado de forma associada à furosemida, porém, não houve consenso quanto ao uso em animais deste estágio, já que seu uso é contra indicado em Humanos com Insuficiência Cardíaca Congestiva, devido baixa quantidade de estudos realizados, que comprovem a segurança da utilização destes fármacos.

O uso da pomada de nitroglicerina, com o objetivo de promover vasodilatação, também não é recomendado pelo consenso por não existirem trabalhos à respeito dos benefícios e malefícios de sua utilização. Porém, quando utilizada, 
ROCHA, R.C. et al. Sopro intermitente em cão: relato de caso. PUBVET, Londrina, V. 8, N. 17, Ed. 266, Art. 1771, Setembro, 2014.

os animais submetidos a ela apresentaram melhora no quadro de Edema Pulmonar.

Outros medicamentos que podem ser benéficos neste estágio são a Dobutamina, que promove um melhor desempenho cardíaco através da ação simultânea com receptores Beta 1 , que promoverão uma melhora na contratilidade cardíaca. A Hidralazina diminui a pós - carga, bloqueando os canais de cálcio, causando vasodilatação arteriolar.

- Classe C2: Nesta classe se encaixam os animais que apresentam sinais clínicos que implicam em tratamento para casa, ou aqueles que já necessitaram de hospitalização para estabilização dos sinais clínicos da DVCD. Estes animais podem ser manejados em casa, com medicações por via oral. As medicações indicadas para esta classe são: Diuréticos ( Ex. Furosemida, Espironolactona), Inotrópicos positivos (Ex. Pimobendam) e IECAS (Enalapril, Benazepril). O diurético é utilizado com o objetivo de prevenir o desenvolvimento de Edema Pulmonar, através do controle do líquido extra celular, diminuindo a pressão dos vasos, diminuindo assim a pré - carga.

- Classe D: Neste estágio estão os animais que apresentam a doença em sua forma mais grave, com sinais clínicos de Insuficiencia Cardíaca e de Remodelamento Cardíaco, e que não respondem mais à terapia padrão.

Alguns animais necessitam de uma estratégia terapêutica avançada ou especializada, a fim de manter a condição clínica de forma estável. Como referido no estágio $C$, os animais deste estágio (Estágio D), são aqueles que necessitam de terapia médica intensiva, com o objetivo de estabilizar sua condição clínica. Posteriormente, estes animais necessitarão de um manejo terapêutico especial, para que os sinais clínicos característicos deste estágio se manifestem, a fim de manter - se uma boa qualidade de vida para estes pacientes. No mais, estes animais são aqueles que estão mais susceptíveis a desenvolverem complicações como por exemplo edema pulmonar e ruptura de cordoalha maior. Este estágio se subdivide em estádio D1, e estágio D2, que serão detalhados a seguir. 
ROCHA, R.C. et al. Sopro intermitente em cão: relato de caso. PUBVET, Londrina, V. 8, N. 17, Ed. 266, Art. 1771, Setembro, 2014.

- Classe D1: Os animais deste estágio são aqueles que apresentam sinais clínicos tão graves de Insuficiencia Cardíaca, que necessitam de hospitalização imediata. É considerado o estágio mais grave da doença. O tratamento para estes animais é semelhante ao protocolo utilizado para os animais da classe C1, visando primordialmente a estabilização hemodinâmica e respiratória do animal. Protocolos terapêuticos não farmacológicos, como ventilação mecânica, drenagem cavitária e sedação são de extrema importância para a manutenção dos animais deste estágio.

A respeito do tratamento, houve consenso na utilização de Furosemida por via intra venosa, com o objetivo de melhorar o quadro respiratório do animal. A dosagem utilizada varia de $1 \mathrm{mg} / \mathrm{kg} / \mathrm{h}$ a $2.3 \mathrm{mg} / \mathrm{kg}$, está deverá ser calculada tendo coo critério base o quadro em que se encontra o animal, e sua resposta ao tratamento. O uso do Pimobendan, caso este ainda não seja utilizado para o tratamento em ambiente extra - hospitalar, a uma dose incial de 0.25 a 0.3 $\mathrm{mg} / \mathrm{kg}$ a cada 12 horas, é importante devido sua função hemodinâmica, e por trazer bons resultados ao ser utilizado em animais em estado grave. Medicações mais agressivas como por exemplo o nitroprussiato de sódio ( 1 $5 \mathrm{ug} / \mathrm{kg} / \mathrm{min})$, a hydralazina $(0.5-2 \mathrm{mg} / \mathrm{kg}$ BID) e a amlodipina (0.05 - 0.1 , $\mathrm{g} / \mathrm{kg}$ SID) podem ser utilizados, porém, devemos nos atentar às alterações causadas por estes fármacos, para evitarmos a ocorrência de hipotensão. Há também a indicação de que caso seja necessária a utilização de fármacos mais agressivos, estes podem ser associados com medicações de efeito inotrópico positivo, como por exemplo o Pimobendan.

- Classe D2: Neste estágio os animais já desenvolveram sinais clínicos graves de Insuficiencia Cardíaca, e já foram submetidos ao tratamento hospitalar, porém, apresentando melhora significativa do quadro, sendo encaminhandos para o tratamento fora do ambiente hospitalar, a base de medicações adminsitradas por via oral.

As medicações indicadas pelo consenso, para o tratamento destes animais são: Furosemida, como diurético utilizado para evitar a ocorrência de edema pulomonar, na dose de $1-2 \mathrm{mg} / \mathrm{kg}$ BID, podendo ser utilizada na dosagem de 
ROCHA, R.C. et al. Sopro intermitente em cão: relato de caso. PUBVET, Londrina, V. 8, N. 17, Ed. 266, Art. 1771, Setembro, 2014.

4 - $6 \mathrm{mg} / \mathrm{kg} / \mathrm{TID}$, dependendo do estado clínico do animal, havendo também a opção de associação com outro diurético, como a Hidroclortiazida ou Espironolactona, visando podencializar a diurese.

Fármacos da classe IECA, podendo ser por exemplo Maleato de Enalapirl na dose de $0.5 \mathrm{mg} / \mathrm{kg}$ BID, e o Pimobendam, na dose de $0.25-0.3 \mathrm{mg} / \mathrm{kg} / \mathrm{BID}$ também podem ser usados.

Outros fármacos que podem ser utilizados para o manejo caseiro dos animais deste estágio são: Digoxina, utilizado como anti arrítmico na dose de 0.22 $\mathrm{mg} / \mathrm{m}^{2}$ BID. Espironolactona, como diurético atuante em outras áreas do néfron, visando potencializar o efeito diurético dos outros fármacos, na dosagem de $0.25-2 \mathrm{mg} / \mathrm{kg}$ BID ou SID, e fármacos broncodilatadores, como Aminofilina utilizada a $10 \mathrm{mg} / \mathrm{kg}$ TID.

Esta classificação demonstra que os fatores de risco para o aparecimento, desenvolvimento e evolução dos sinais clínicos da DVCD, e de Insuficiência Cardíaca, estão relacionados diretamente com as características do paciente, como por exemplo, fatores genéticos e idade. [22, 24].

O tratamento para a DVCD é paliativo, e objetiva estabilizar e minimizar as lesões e alterações sistêmicas, causadas pela Insuficiencia resultante do refluxo valvar, decorrentes do prolapso. Conclui -se então, que o tratamento se baseia em medicamentos Inibidores da Enzima Conversora de Angiotensina (IECA), Diuréticos, Bloqueadores do Canal de Cálcio e Reguladores de Vasoconstrição.

Os fármacos da classe IECA (Por exemplo Enalapril, Benazepril), são os mais comumente usados nos casos de DVCD. Muitos estudos, duplo - cegos, multicêntricos, e randômicos demonstraram que os fármacos da classe IECA melhoram a qualidade de vida, aprimoram a tolerância ao exercício físico, e aumentam a expectativa de vida dos animais com insuficiência cardíaca [23]. Há evidencias convincentes de que a supressão do sistema renina angiotensina aldosterona, com esta classe farmacológica, quando utilizados simultaneamente com outros fármacos, melhoram o estado clínico do animal, além de proporcionar melhores resultados no tratamento fora do ambiente 
ROCHA, R.C. et al. Sopro intermitente em cão: relato de caso. PUBVET, Londrina, V. 8, N. 17, Ed. 266, Art. 1771, Setembro, 2014.

hospitalar.[17]. Os fármacos IECA produzem uma vasodilatação sistêmica, favorecendo a hemodinâmica do animal, diminuindo a retenção de líquidos, agindo diretamente na regulação da pressão e na prevenção do desenvolvimento de edema pulmonar, além de estabilizar os processos progressivos de remodelamento cardíaco [24].

A furosemida, um diurético de ação em alça de Henle, é um dos fármacos mais comumente utilizados no tratamento da DVCD, em conjunto com os fármacos IECA. A furosemida possui uma ação potente, diminuindo o volume total de líquido circulante, consequentemente diminuindo a pressão em átrio esquerdo. Porém, a utilização primária deste fármaco objetiva a prevenção da ocorrência de Edema Pulmonar Cardiogênico [26].

A espironolactona, é um fármaco muito utilizado como suporte ao tratamento convencional da Insuficiência Cardíaca. Sua ação antagonista competitiva da aldosterona, auxilia na retenção de Potássio, que é excretado em excesso quando há utilização de diuréticos como a Furosemida [15].

A adição da Espironolactona ao tratamento convencional da insuficiência cardíaca reduziu o número de animais mortos por complicações cardíacas, renais, ou das duas em conjunto [27].

\section{Relato de Caso:}

O animal Mike, Shih Tzu, Macho, três anos, deu entrada no Hovet Ung apresentando como queixa principal um episódio de sincope, após agitação. A proprietária, relatou também que o animal se apresentava prostrado, com secreção ocular purulenta bilateral, com possível quadro de pré síncope. A proprietária referiu também que o estado prostrado em que o animal se apresentava, teve início aproximadamente quatro dias antes do episódio de sincope. Foi relatado também que o animal apresentava cansaço fácil, engasgos e ruídos respiratórios durante agitação, refere sincope, porém nega relutância ao praticar exercícios físicos. À auscultação, o animal apresentou arritmia sinusal, sopro com foco em valva mitral, de grau 4 / 6, em momentos de 
ROCHA, R.C. et al. Sopro intermitente em cão: relato de caso. PUBVET, Londrina, V. 8, N. 17, Ed. 266, Art. 1771, Setembro, 2014.

taquicardia, mas que não se apresentava audível em momentos de frequência cardíaca mais baixas, campos pulmonares limpos à auscultação. Ao exame físico as mucosas apresentavam - se levemente hipocoradas, hidratação adequada, Palpação abdominal sem alterações. À realização do Ecodopplercardiograma, foi evidenciada a presença de uma insuficiência valvar mitral, caracterizado por um "mosaico" na imagem gerada, que demonstra uma circulação anormal no átrio esquerdo, correspondente ao sangue que refluía do ventrículo para o átrio, como já demonstrado na figura 5. Porém, diferentemente dos sopros convencionais, este, não estava presente em todas as contrações cardíacas. O animal não apresentava nenhuma outra alteração evidente no ecodopplercardiograma, além do prolapso valvar e do refluxo sanguíneo decorrente desta condição.

\section{Discussão:}

Durante a realização do Ecodopplercardiograma, concluímos que a o fator causador da intermitência do refluxo sanguíneo, era a arritmia sinusal que o animal apresentava, pois o refluxo se tornava evidente quando havia taquicardia, porém, em momentos de frequência cardíaca mais próximas do valor normal, ou em momentos de bradicardia, o refluxo não era mais visível.

Apesar da condição apresentada, não foram adotados protocolos terapêuticos, pois como já discutido neste trabalho, este animal é classificado classe B1, pois apresenta uma regurgitação valvar, porém ainda não apresentando remodelamento de camara cardíaca, fator este considerado principal como diferencial para o inicio do tratamento de insuficiência cardíaca, mesmo que o animal apresente sinais clínicos, como a síncope em momentos de agitação, quando o refluxo valvar se tornava importante, possivelmente, diminuindo de forma aguda o débito cardíaco. Outro fator definitivo para a decisão de não iniciar protocolo terapêutico foi o desaparecimento das manifestações clínicas causadas pelo baixo débito cardíaco, como a síncope, que cessaram após o aumento da massa muscular do animal, a qual pode ter diminuído a 
ROCHA, R.C. et al. Sopro intermitente em cão: relato de caso. PUBVET, Londrina, V. 8, N. 17, Ed. 266, Art. 1771, Setembro, 2014.

sobrecarga cardíaca, consequentemente diminuindo o refluxo valvar. A conduta correta a ser realizada seria a observação do animal, para acompanhamento da progressão da Insuficiencia Valvar, para iniciar o protocolo de tratamento adequado quando houvesse necessidade. Porém, a proprietária não compareceu aos retornos, impossibilitando o acompanhamento clínico por parte da equipe responsável, tornando inviável a descrição e documentação de uma possível progressão e evolução do quadro, de forma atípica, ou de forma diferente da mais comumente observada em sopros característicos.

\section{Referencias Bibliográficas:}

1: Ware, A. W., Doenças do Sistema Cardiovascular. In: NELSON, Richard W.; COUTO, C Guilhermo. Manual de Medicina Interna de Pequenos Animais. 2. ed. Rio de Janeiro: Elsevier, 2006. p. 95-103.

2: Pedersen, H. D., Haggstrom, J. Mitral Valve prolapsed in the dog: A model of mitral valve prolapsed in man. Cardiovascular Research 47, 2000, p. $234-243$.

3: Hayek, E., Gring, C. N., Griffin, B.P. Mitral Valve Prolapse, Lancet 2005; 365: 507-18.

4:Devereux RB. Recent developments in the diagnosis and management of mitral valve prolapse. Curr Opin Cardiol 1995;10:107-116.

5: Braunwald, E., Valvular heart disease. In: Braunwald E, editor, Heart disease. A textbook of cardiovascular medicine, 5th ed, Philadelphia: W.B. Saunders, 1997, pp. 1007-1072.

6: Lam, J H C, et al, Circulation. J Vet Med, 1970, 41, p. 440-449.

7: Perloff, J K, and Roberts, W C, Circulation. J Vet Med, 1972, 46, p. 219-227.

8: Haggstrom J, Kvart C, Pedersen HD. Acquired valvular disease. In: Ettinger SJ, Feldman EC, eds. Textbook of Veterinary Internal Medicine, 6th ed. St Louis: Elsevier; 2005:1022-1039.

9: Pedersen HD, Kristensen BO, Norby B, Lorentzen KA. Echocardiographic study of mitral valve prolapse in Dachsunds. J Vet Med A 1996;43:103-110.

10: Borgarelli, M., Savarino, P., S. Crosara. et all, Survival Characteristics and Prognostic Variables of Dogs with Mitral Regurgitation Attributable to Myxomatous Valve Disease, J Vet Intern Med 2008;22:120-128.

11: Thrusfield, M. V., Aitken, C. G. G., Darke, P. G. G. Observations on breed and sex in relation to canine heart valve incompetence. J. Samil. Anim. Pract., v. 26, 1985.

p. $709-717$.

12: Atkins, C. E. Acquired valvular insufficiency in: MILLER, M.S., TILLEY, L.P. Manual of canine and feline cardiology. 2. ed. Philadelphia: W.B. Saunders, 1995. p. $129-144$. 
13: Buchanan, J.W. Chronic valvular disease (endocardiosis) in dogs. Adv. Vet. Sci. Comp. Med., v 21, 1997. p $75-106$

14: A. Ware, Wendy. Doenças do Sistema Cardiovascular. In: NELSON, Richard W.; COUTO, C Guilhermo. Manual de Medicina Interna de Pequenos Animais. 4. ed. Rio de Janeiro: Elsevier, 2010. p. 52-71.

15: P. M. Pereira, A. A. Camacho, H. A. Morais. Tratamento da Insuficiência Cardíaca com Benazepril em cães com cardiomiopatia dilatada e endocardiose. Arquivo Brasileiro de Medicina Veterinária e Zootecnia, v. 57, supl. 2, p. 141 - 148, 2005.

16: C. Kvart et. Al. Efficacy of Enalapril for Prevention of Congestive Heart Failure in Dogs with Myxomatous Valve Disease and Assymptomatic Mitral Regurgitation. J. Vet. Intern. Med. $2002 ; 16$, p. $80-88$

17: Pesinato, R. M. et al. A expertise Clássica da ausculta cardíaca para o diagnóstico do prolapso da valva mitral. Ver Bras Clin Med. São Paulo, 2012 mai-jun;10(3):222-5

18: Camacho, A., Mucha, C., José. Sistema Circulatório: Semiologia do Sistema Circulatório de Cães e Gatos. In: FEITOSA, Franciso Leitoso. Semilogia Veterinária: A arte do Diagnóstico. 2a São Paulo: Roca, 2008. Cap. 6, p. 282-311.

19: R. Suzuky, H. Matsumoto, T. Teshima, et al. Influence of Heart rate on myocardial function using two - dimensional speckle - tracking echocardiography in healthy dogs. Journal of veterinary cardiology, 2013 (15), p. $139-146$.

20: Schober, K. E., Hart, T. M., Stern, J. A. et. Al. Detect of Congestive Heart Failure in Dogs By Doppler Cardiography. J. Vet. Intern. Med. 2010;24 p. 1358 - 1368.

21: Atkins, C., et al. Guidelines for the Diagnosis and Treatment of Canine Chronic Valvular Heart Disease. ACVIM consensus statement. J. Vet. Intern. Med. 2009;23: p. 1142 - 1150.

22: Pouchelon, J. - L., et al. Effect of Benazepril on Survival and Cardiac Events in Dogs With Asymptomatic Mitral Valve Disease: A Retrospective Study of 141 Cases. J. Vet. Intern. Med. 2008;22. P. $905-914$.

23: Atkins, C. E., Haggstrom, J. Pharmacologic Management of Myxomatous Mitral Valve Disease In Dogs. Journal of Veterinary Cardiology, 2012;14. P. 165 - 184.

24: Ferasin, L. et al. Risk Factors for Coughing in Dogs with Naturally Acquired Myxomatous Mitral Valve Disease. J. Vet. Intern. Med. 2013;27. P. $286-292$

25: Suzuki, S. et al. The effect of Furosemide on Left Atrial Pressure in Dogs with Mitral alve Regurgitation. J. Vet. Intern. Med. 2011;25. P. $244-250$.

26: Lefebvre, H. P., et al. Safety Os Spironolactone In Dogs With Chronic Heart Failure because of Degenerative Valvar Disease: A Population - Based, Longitudinal Study. J. Vet. Intern. Med. 2013, p. 1 - 9.

Figura 1: Camaras e Valvas Cardíacas. Disponível em: http://saude.hsw.uol.com.br/coracao1.htm, por Dr. Carl Bianco, M.D, acessado em $12 / 10 / 2013$. 
Figura 2: Valva Mitral Prolapsada. Disponível em:

http://www. hopkinsmedicine.org/healthlibrary/conditions/cardiovascular diseases/mitral valve prolapse 85,P00229/, acessado em 12/10/2013.

Figura 3: Camaras cardíacas evidenciadas por Ecodopplercardiograma. Imagem cedida pelo Instituto Naya de Cardiologia Veterinária.

Figura 4: Prolapso Valvar evidenciado por Ecodopplercardiograma. Imagem cedida pelo Instituto Naya de Cardiologia Veterinária.

Figura 5: Mosaico em átrio esquerdo causado pelo refluxo sanguíneo proveniente do prolapso valvar. Imagem cedida pelo instituto Naya de Cardiologia Veterinária. 